\title{
A new fundamental type of conformational isomerism
}

\author{
Peter J. Canfield ${ }^{\S \dagger \perp}$, Iain M. Blake ${ }^{\dagger}$, Zheng-Li Cai ${ }^{\dagger}$, Ian J. Luck ${ }^{\dagger}$, Elmars Krausz $^{\ddagger}$, Rika Kobayashi ${ }^{\Phi \S}$, \\ Jeffrey R. Reimers ${ }^{\S \#^{*}}$ and Maxwell J. Crossley ${ }^{\dagger *}$ \\ $\S$ International Centre for Quantum and Molecular Structures, Shanghai University, Shanghai \\ 200444, China \\ † School of Chemistry, The University of Sydney, NSW 2006, Australia \\ $\perp$ OraInnova, PO box 968 Darlinghurst NSW 1300 Australia \\ $\ddagger$ Research School of Chemistry, The Australian National University, Canberra, ACT 0200, \\ Australia \\ If Australian National University, Leonard Huxley Bldg. 56, Mills Rd, Canberra, ACT, 2601, \\ Australia \\ \# School of Mathematical and Physical Sciences, University of Technology Sydney, NSW 2007 \\ Australia \\ * email: Maxwell.crossley@sydney.edu.au, reimers@shu.edu.cn, jeffrey.reimers@uts.edu.au
}

\begin{abstract}
:
Isomerism is a fundamental chemical concept, reflecting the fact that the arrangement of atoms in a molecular entity has a profound influence on its chemical and physical properties. Here we describe a previously unclassified fundamental form of conformational isomerism through four resolved stereoisomers of a transoid (BF)O(BF)-quinoxalinoporphyrin. These comprise two pairs of enantiomers that manifest structural relationships not describable within existing IUPAC nomenclature and terminology. They undergo thermal diastereomeric interconversion over a barrier of $104 \pm 2 \mathrm{~kJ} \mathrm{~mol}^{-1}$, which we term "akamptisomerisation". Feasible interconversion processes between conceivable synthesis products and reaction intermediates were mapped out by densityfunctional theory calculations, identifying bond-angle inversion (BAI) at a singly bonded atom as the reaction mechanism. We also introduce the necessary BAI stereodescriptors parvo and amplo. Based on an extended polytope formalism of molecular structure and stereoisomerisation, BAI-driven akamptisomerisation is shown to be the final fundamental type of conformational isomerisation.
\end{abstract}


The dynamic nature of molecules plays a critical role in determining both their macroscopic and microscopic properties. In the case where the molecular constitution and connectivity remains unchanged, this dynamic behaviour is termed conformational isomerism.

Isomerism is a key concept in chemistry, and understanding the relationships that exist between different isomers - compounds made up of the same atoms, but arranged in different manners - is not only of fundamental interest but also holds vast structural and functional implications. Constitutional isomers, for example, present the same general formula but their atoms are held together through different chemical bonds, making them distinct compounds. In contrast, stereoisomers feature the same connectivities but their atoms are arranged differently. They result from the dynamical nature of molecules, and can be further divided into sub-categories: configurational isomers are interconverted over large energy barriers - this is the case for $(E / Z)$ isomers of alkenes - and are easy to isolate under ambient conditions, whilst conformational isomers interconvert over low barriers — such as a rotation around a single bond - which renders their isolation difficult. Figure 1 provides an overview of key IUPAC terms ${ }^{1}$ and current practice ${ }^{2-10}$ involved in describing isomerism, emphasising stereoisomerism and in particular conformational isomerism; for reference, a full list of relevant terms and their formal definitions is provided in Supporting Information Section 1.

In some cases, specific circumstances can lead conformational isomers to have unusually high interconversion barriers, rendering them isolable. The first of these instances to be discovered was the unexpected bond-rotation-based phenomenon, called atropisomerism, reported in $1914^{11,12}$ and described in $1922^{13}$ and $1933^{14}$. In 1961, similar isolable conformational isomerisation involving pyramidal inversion was discovered in phosphorus compounds ${ }^{15}$, and then in isolable nitrogen compounds in $1968^{16}$. Are there other fundamental forms of conformational isomerisation remaining unidentified? The polytope formalism ${ }^{17-25}$ (introduced in detail in the Supplementary Information Section 1b) has been developed to understand stereoisomerisation processes in molecules containing atoms with high coordination numbers, leading to identification of possible conformational isomers, but such systematic analysis has yet to be applied to low-coordinate species and has not previously been used to describe internal rotation as a reaction pathway.

We show how the polytope formalism can be extended to include bond rotations and provide a comprehensive description of the fundamental stereoisomerism processes for each coordination number. Applying this to the lower coordinate systems, we found an unrecognised fundamental type of conformational isomerism that, for example, could be produced by a bond-angle inversion (BAI) reaction mechanism about centres of the form $M_{1}-X-M_{2}$ (where the central atom $X$ exhibits a bent geometry and $\mathrm{M}_{1}$ and $\mathrm{M}_{2}$ are any atoms) linked by only single bonds. Isolable compounds distinguished by only this feature have not previously been synthesised, although the process has been envisaged as occurring at linkages inside polymeric systems ${ }^{26}$. We make isolable compounds by encapsulating $\mathrm{B}-\mathrm{O}-\mathrm{B}[\mathrm{as}(\mathrm{BF}) \mathrm{O}(\mathrm{BF})]$ inside a porphyrin macrocycle.

No existing IUPAC terminologies ${ }^{1}$ can describe the relationships between the compounds synthesised. We describe these stereoisomers as akamptisomers and their process of interconversion as akamptisomerisation. In Figure 1, the listed standard IUPAC nomenclature has been extended to include this new process. Also, we introduce the new stereodescriptors parvo and amplo for naming akamptisomers and related structures, including classification of transition-state structures and some as yet undiscovered structural possibilities.

\section{Results and discussion}


Generalised polytope formalism for conformational isomerism. In Figure 1, the relationships between existing definitions of isomerisation (according to the IUPAC ${ }^{1}$ ) are depicted graphically using a polytope model extended to include internal rotations as well as vibrations which occur in lower-coordinate systems. Full details and extensive discussion is provided in Supporting Information Section 1, including a current listing of all relevant IUPAC definitions. While the polytope formalism has been instrumental in understanding complex processes in molecules featuring high-coordinate centres, the application of these ideas to lower-coordinate species has not previously been a priority. Well-known useful results are immediately obtained, however, such as the demonstration that pyramidal molecules like ammonia can invert only through either trigonal planar or T-shaped transition states, thus defining reaction landscapes. Similarly, the formalism indicates that rotation about a bond leads to well-known processes such as (E/Z)-stereoisomerisation and anti and gauche conformers interconverting through eclipsed structures. For bent triatomic molecules such as water, the formalism indicates that the only available process involves inversion of the $\mathrm{H}-\mathrm{O}-\mathrm{H}$ bond angle. In this case, BAI results in an equivalent molecule, so the process appears uninteresting. It is nevertheless a fundamental process as it cannot universally be described in terms of any combination of other fundamental processes; the question of interest concerns whether or not interesting chemistry can be found characterised only by BAI. Here, a critical distinction concerns chemistry involving single bonds versus multiple bonds, something already inherent in IUPAC nomenclature that is demanded by the polytope formalism, and the interaction between rotation about bonds and BAI in each case.

In doubly bonded molecules such as substituted imines $C\left(\mathrm{R}^{1} \mathrm{R}^{2}\right)=\mathrm{N}\left(\mathrm{R}^{3}\right)$, rotation about a bond and BAI provide alternate concerted mechanisms for $(E / Z)$ stereoisomerisation. ${ }^{5,27-45}$ The bond-rotation transition state in non-planar and naively involves $\mathrm{sp}^{2}$ to $\mathrm{sp}^{3}$ re-hybridisation at the $\mathrm{N}$ atom, whereas the planar BAI transition state involves $\mathrm{sp}^{2}$ to $\mathrm{sp}$ re-hybridisation at the $\mathrm{N}$. However, as the reaction products are the same, there has been no need for unique product or process names, with all isolated compounds amenable to standard nomenclature. As in the example of water discussed above, BAI in singly bonded systems has not been shown to lead to interesting chemistry. Consequently, in modern treatises on stereochemistry ${ }^{2-10}$, BAI is mentioned as a reaction mechanism but not as a fundamental descriptor of conformational isomerism ${ }^{5}$, and terminology specifically associated with it is not described in the IUPAC "Gold Book"1.

In comparison, processes involving atoms with only single bonds have been regarded as being uninteresting, but the discovery of atropisomerism ${ }^{11-14}$ — isolable compounds differentiated only by rotation about single bonds - necessitated the introduction of a different chemical nomenclature to that used for doubly bond systems (Figure 1). The extended polytope formalism indicates that the same situation applies for BAI, with the parallel phenomenon - akamptisomerism — remaining undiscovered. Rotation about single bonds does not change hybridization whereas BAI involves sp $^{3}$ to sp re-hybridisation at the central atom. The observation of akamptisomerisation demands the synthesis of isolable compounds distinguishable through BAI. Note, however, that observed interconversion of akamptisomers is not an essential feature, and that whilst BAI must be a conceivable mechanism for any observed interconversion, it will usually not be the only mechanistic possibility. The challenge is that, for akamptisomers to be isolable, all possible interconversion mechanisms must have sufficiently high barriers. The alternate process of greatest relevance involves the combination of two fundamental atropisomerism processes (torsions) occurring about each of the bonds involved in the BAI.

Compound design and synthesis. If the $\mathrm{H}$ atoms in water are replaced by three-dimensional chemical groups as in $\mathrm{M}_{1}-\mathrm{X}-\mathrm{M}_{2}$, then the relationship between the end groups is not preserved during BAI. Even so, isolable compounds are not usually produced. Consider for example, 
methoxyethane shown in Figure 2a. A BAI reaction would invert the $\mathrm{C}-\mathrm{O}-\mathrm{C}$ angle to produce what is a higher-energy structure, but this would undergo a barrierless or low-barrier relaxation involving the torsional motions about the two $\mathrm{C}-\mathrm{O}$ bonds to regenerate the original structure, with no new isomer to be isolated. In the known examples of BAI reactions, these processes relate to multiply bonded systems with moderate to large barriers towards bond rotation, allowing (E/Z)stereoisomerisation to produce isolable compounds. To observe akamptisomerism, external restraints must be introduced that prevent unwanted torsional motions about single bonds. The anchoring of both ends of the inverting group within a macrocyclic compound is a way to achieve this goal.

The porphyrin macrocycle provides a very versatile scaffold, exploited by Nature and chemists alike, chiefly due to its capacity to coordinate many elements in its central cavity. Brothers et al. have developed the somewhat unusual class of porphyrin and related macrocycle derivatives featuring two inner coordinated boron atoms ${ }^{46-56}$ in a 1,3-difluoro- $1 \lambda^{4}, 3 \lambda^{4}$-diboroxan-1,1,3,3-tetrayl group, abbreviated $(\mathrm{BF}) \mathrm{O}(\mathrm{BF})$, that in porphyrins has each boron bonded in a transoid fashion to two adjacent pyrrolic nitrogen atoms. This, in principle, facilitates an encapsulated $\mathrm{B}-\mathrm{O}-\mathrm{B}$ linkage that could lead to isolable akamptisomers. Distinguishable isomers could be produced, for example if one or both positions occupied by the fluorines are asymmetrically substituted akin to the methoxyethane example from Figure 2a.

Indeed, such a porphyrin has been synthesised by Belcher et al. ${ }^{52}$ featuring phenyl and hydroxyl boron substituents. It is shown in Supplementary Table 14 (there named "(amplo,parvo)$\mathrm{B}(\mathrm{OH}) \mathrm{OB}(\mathrm{Ph}) \mathrm{ttp}$ ”) and sketched in Figure 2b. To envisage BAI with this species, the $\mathrm{O}$ atom must be pushed through the middle of the macrocyclic plane. If this could happen the same way as methoxyethane relaxes (Figure 2a), then the structure produced would be that indicated in Figure 2b. However, for this to occur, the bonds in the macrocycle must be broken and reformed differently, with in effect the whole $\mathrm{B}(\mathrm{OH}) \mathrm{OB}(\mathrm{Ph})$ unit effectively pushed through the porphyrin ring. At the simplest level, torsional motions undoing the BAI would therefore be thought to be completely infeasible.

However, by closely coupling the two torsional motions together, a potentially feasible reversal can be envisaged, as sketched in Figure 2c for the difluoro analogue. It involves no bond breakage with only the $\mathrm{O}$ atom being pushed through the macrocycle ring, producing a transition state of $\mathrm{C}_{2}$ symmetry. As by assumption $\mathrm{B}-\mathrm{O}-\mathrm{B}$ bending is not involved in this process, its angle is required to remain of the order of the tetrahedral angle. This bent configuration forces the oxygen to one side of the macrocyclic ring as it passes through, inducing significant steric repulsions. Supporting Information Section 6.c.ii shows that competition between this steric repulsion energy and the intrinsic BAI bending energy determines the reaction mechanism.

For the molecule shown in Figure 2c, B3LYP and coupled-cluster calculations (see Methods) indicate that the steric energy is too great, making the indicated coupled-torsion structure much higher in energy than that for the BAI transition state. Any attempt at a double-torsion reaction will therefore collapse to the linear BAI transition state, a singularity point in the definition of the double-torsion process. Recognizing this singularity, the mathematically rigorous polytope formalism views the double-torsion and BAI processes as being fundamentally different; one cannot generally view BAI inside a macrocycle as a composite isomerisation built by combining two atropisomerisations. For this example, coupled torsional motions cannot emulate or undo the BAI reaction and hence the resulting akamptisomers are expected to be isolable. 
One could anticipate that, for the compound synthesised by Belcher et al. ${ }^{52}$ shown in Figure 2b, BAI would produce a second distinct, isolable, stereoisomer (it's akamptisomer). However, in this specific example, only the initial structure has been isolated, with calculations indicating that the akamptisomer has increased steric interactions between the phenyl and the porphyrin rings ${ }^{52}$, rendering it thermodynamically uncompetitive ${ }^{57}$.

An alternate concept retaining the symmetrical fluorine substitution found in many synthesised compounds but still leading to distinct structures produced by BAI is to asymmetrically substitute the macrocyclic ring. This process is illustrated in Figure 2d. In this case, akamptisomers are differentiated by their interactions with the asymmetric substitution, with coupled torsional motions again unable to reverse the BAI owing to the steric clashes highlighted in Figure 2c. As the asymmetric substitution could occur at locations well removed from the $(\mathrm{BF}) \mathrm{O}(\mathrm{BF})$ group, by using this strategy it is possible to guarantee only small energy differences between the akamptisomers. While energy differences may be small, the added substituents could nevertheless produce significant differences in the isomer shape and other exploitable properties, opening a new chemical dimension.

Matching macrocycle cavity size and inner-ligand size is important. Given the wide choices available for macrocycle, its inner coordinated bridge group, and feasible symmetries, a wide range of akamptisomeric pairs could one day be synthesised.

Seeking to isolate akamptisomers, we have pursued the ring substitution approach, performing a well-known ${ }^{46-53,58}$ difluorodiboroxanation synthesis reaction (Figure 3) on the $\beta, \beta$ '-pyrrolic fused quinoxalinoporphyrin 1 which could give four racemates $2,3,4$, and 5 , each comprising enantiomers a and $\mathbf{b}$. All 8 compounds would have very different 3-dimensional shapes owing to their chirality and ring distortion associated with the $(\mathrm{BF}) \mathrm{O}(\mathrm{BF})$ substitution, accentuated by the large size of the quinoxalino group. Compounds $\mathbf{2}$ and $\mathbf{3}$ are transoid compounds related by BAI, whilst $\mathbf{4}$ and $\mathbf{5}$ are similarly related cisoid species. Previous syntheses embedding (BF) $\mathrm{O}(\mathrm{BF})$ inside porphyrin macrocycles have yielded products akin to the transoid species only ${ }^{46-53}$, with no chiral synthesis being attempted and no pairs of akamptisomers isolated. However, analogous cisoid species are observed when reactions are performed on corroles instead of porphyrins ${ }^{55,58-60}$.

Treatment of quinoxalinoporphyrin $\mathbf{1}^{61-63}$ with excess $\mathrm{Et}_{2} \mathrm{O} \cdot \mathrm{BF}_{3} / \mathrm{NEt}_{3}$ in $\mathrm{CH}_{2} \mathrm{Cl}_{2}$ solution at $50^{\circ} \mathrm{C}$ and subsequently with $1 \mathrm{M}$ aqueous $\mathrm{NaOH}$ afforded only the racemates 2 and $\mathbf{3}$ in $39 \%$ and $40 \%$ yields, respectively. A wide range of ${ }^{19} \mathrm{~F},{ }^{13} \mathrm{C}$ and ${ }^{1} \mathrm{H}$ nuclear magnetic resonance (NMR) data including correlated ${ }^{13} \mathrm{C}$ and ${ }^{1} \mathrm{H}$ spectra was obtained (see Supplementary Information Section 3) that unambiguously allow the structural assignment of the products as $\mathbf{2}$ and $\mathbf{3}$. Alternatively, chiral HPLC of the reaction mixture gave, in order of elution, enantiopure $\mathbf{2} \mathbf{b}, \mathbf{2} \mathbf{a}, \mathbf{3 a}$, and $\mathbf{3 b}$, distributed $25.0 \pm 1 \%$ into each fraction, see Methods and Supporting Information Sections 2 and 7.

Supplementary Video 1 shows animations of the transoid species $\mathbf{2 a}, \mathbf{2} \mathbf{b}, \mathbf{3 a}$ and $\mathbf{3} \mathbf{b}$ to highlight their subtle stereochemical differences. All four have the same local coordination patterns around each boron atom and are therefore expected to be of similar energy, with only the distant quinoxalino group providing differentiation between 2 and 3. Buckling of the porphyrin results in one half of the porphyrin macrocycle with its coordinated boron remaining largely planar, whilst the other half distorts to present a strikingly out-of-plane boron; yellow highlighting in Figure 3 indicates rings, attached to and coplanar with, the in-plane boron atom. Supplementary Video 2 similarly shows the unidentified cisoid species $\mathbf{4 a}, \mathbf{4 b}, \mathbf{5 a}$ and $\mathbf{5 b}$. 
Isomerisation investigations. In the solid form, all four fractions were stable at ambient temperatures, but in solution (either $\mathrm{CDCl}_{3}$ as used for NMR or 99:1 n-hexane:2-propanol as used for HPLC), heating induced the following specific diastereomeric interconversions:

$$
2 \mathbf{b} \rightleftharpoons 3 \mathbf{b} \text { and } 2 \mathbf{a} \rightleftharpoons 3 \mathbf{a}
$$

In Supplementary Information Section 4, the rate constants for these processes are determined using ${ }^{19} \mathrm{~F}$ NMR, leading to the conclusion that the free-energy changes associated with each reaction are $\Delta G=0.0 \pm 0.2 \mathrm{~kJ} \mathrm{~mol}^{-1}$, while the activation energies are $\Delta G^{\ddagger}=104 \pm 2 \mathrm{~kJ} \mathrm{~mol}^{-1}$. No racemisation (interconversion between $\mathbf{2} \mathbf{b}$ and $\mathbf{2 a}$ or between $\mathbf{3 b}$ and $\mathbf{3 a}$ ) was observed under these conditions.

To show the relationships between the conceived compounds 2-5, density-functional theory (DFT) calculations at the B3L YP/6-31+G* level were performed, seeking stable isomers corresponding to 2-5 and transition states for BAI and other possible unimolecular processes, as well as for possible bimolecular processes, that could interconvert them. Full details are given in Supplementary Information Section 6, with optimized coordinates provided in Supporting Data.

Stable structures were predicted for 2-4 but only intermediary structures for $\mathbf{5}$. Calculated potentialenergy surfaces depicting BAI as a function of the generalised $\mathrm{B}-\mathrm{O}-\mathrm{B}$ angle are shown in Figure 4 for $\mathbf{2 a} \rightleftharpoons 3 \mathbf{a}$ (or $\mathbf{2} \mathbf{b} \rightleftharpoons \mathbf{3 b}$ ) equilibration and for the relaxation $5 \mathbf{a} \rightarrow \mathbf{4 a}$ (or $5 \mathbf{b} \rightarrow \mathbf{4 b}$ ). The calculated free-energy difference $\Delta G_{3}-\Delta G_{2}$ is $0.1 \mathrm{~kJ} \mathrm{~mol}^{-1}$, in good agreement with the observed value of 0.0 $\pm 0.2 \mathrm{~kJ} \mathrm{~mol}^{-1}$; also, $\Delta G_{4}-\Delta G_{2}$ is $30 \mathrm{~kJ} \mathrm{~mol}^{-1}$, indicating that 4 would not be formed in measurable yield if synthesis was thermodynamically controlled, with $\mathbf{5}$ appearing as a very high energy intermediary at $\Delta G_{5}-\Delta G_{2}$ of the order of $90 \mathrm{~kJ} \mathrm{~mol}^{-1}$.

Table 1 details calculated and observed activation free energies for various anticipated unimolecular or bimolecular processes expected either under both the synthetic reaction conditions or for isolated compounds in solution. These quantities reveal the chemical connectivities linking all 8 isomers shown in Figure 3, considering possible reactions showing: i) $\mathrm{B}-\mathrm{O}-\mathrm{B}$ BAI, ii) (BF)O(BF) rotation, iii) $\mathrm{F}^{-}$loss, iv) $\mathrm{HNEt}_{3}{ }^{+}$catalysed $\mathrm{F}^{-}$loss, v) $\mathrm{BF}_{3} \cdot \mathrm{NEt}_{3}$ catalysed $\mathrm{F}^{-}$loss, vi) uncatalysed $\mathrm{F}^{-} \mathrm{S}_{\mathrm{N} 2}$, and vii) $\mathrm{F}^{-}$loss with counterfacial addition. Only one low-energy process is predicted, the $\mathrm{B}-\mathrm{O}-\mathrm{B}$ BAI $\mathbf{2} \mathbf{a} \rightleftharpoons 3 \mathbf{a}$ (or $\mathbf{2} \mathbf{b} \rightleftharpoons \mathbf{3} \mathbf{b}$ ) equilibration depicted in Figure 4 , for which the calculated activation free energy change of $108 \mathrm{~kJ} \mathrm{~mol}^{-1}$ is in excellent agreement with the observed value of $104 \pm 2 \mathrm{~kJ} \mathrm{~mol}^{-1}$ (Table 1). BAI motion along the reaction coordinate is depicted in Supplementary Video 3, whilst Supplementary Video 4 shows the corresponding vibrational mode (see Supplementary Information Section 1 on the polytope formalism). All other reactions between compounds $\mathbf{2}$ and $\mathbf{3}$ are calculated to have much higher activation energies of at least $180 \mathrm{~kJ} \mathrm{~mol}^{-1}$ and are hence unlikely to contribute to the observed process. Figure 4 also depicts barrierless $5 \mathbf{a} \rightarrow 4 \mathrm{a}$ (or $5 \mathbf{b} \rightarrow 4 \mathbf{b}$ ) BAI relaxation for the cisoid compounds. For isomerisation of $\mathbf{4 b}$, the reaction calculated to have the lowest-energy barrier, $82 \mathrm{~kJ} \mathrm{~mol}^{-1}$, is for an uncatalysed $\mathrm{F}^{-} \mathrm{S}_{\mathrm{N} 2}$ reaction converting 4 to 2 or $\mathbf{3}$; this barrier is sufficiently low for any $\mathbf{4}$ produced during synthesis to isomerise. Hence $\mathbf{4}$ is predicted to be kinetically unstable as well as thermodynamically disfavoured.

Chemical terminology and nomenclature. New terms are required to describe the observed isomerisation processes involving $\mathbf{2}$ and $\mathbf{3}$, as well as those that may also be demonstrated between suitable analogues of $\mathbf{4}$ and $\mathbf{5}$. Whilst many reactions may be envisaged that interconvert $\mathbf{2 a} \rightleftharpoons \mathbf{3 a}$ (or $\mathbf{2} \mathbf{b} \rightleftharpoons \mathbf{3 b}$ and so on), the only accessible and general process available is $\mathrm{B}-\mathrm{O}-\mathrm{B}$ BAI. Hence we describe the (potentially) isolable diastereomeric pairs (2a, 3a), (2b, 3b), (4a, 5a) and (4b, 5b) as akamptisomers and the interconversion process between them as akamptisomerisation (from Greek 
akamptos meaning "inflexible, unbending”), this process being the angle-bending analogue of atropisomerism (from Greek atropos meaning “without turning”).

Also, no standard IUPAC descriptors ${ }^{64}$ exist for akamptisomer names, although for some of these molecules existing descriptors could be utilised. If a clear macrocyclic plane is defined by the surroundings, as is the case for $\mathbf{2 - 5}$, the $\alpha$ and $\beta$ descriptors specifying locations of atoms above and below this plane could, in this case, specify all akamptisomers ${ }^{65}$. However, such a notation is neither general, intuitive nor revealing, has only limited scope, and is not currently applied in standard naming protocols (see Supplementary Information Section 8). We propose universal stereodescriptors for BAI nomenclature, enabling easy implementation within computerised naming schemes. These provide general molecular structural elements that are intuitively descriptive, and can also be applied to BAI systems involving double bonds, if required.

The essential features of BAI in a macrocyclic environment are (i) angle bending around a bicoordinate atom $X$ linked to atoms $M_{1}$ and $M_{2}$, forming an inner $M_{1}-X-M_{2}$ unit ((BF)O(BF) for 25), and (ii) this unit is constrained by bonds to its external environment (macrocyclic ligand, polymer chain, etc.). Both $\mathrm{M}_{1}$ and $\mathrm{M}_{2}$ may connect to their environment by more than one bond (e.g., two $\mathrm{B}-\mathrm{N}$ bonds each for 2-5), the simplest representation of which is a single vector that averages all of the actual bond vectors. These vectors end at points named $E_{1}$ and $E_{2}$ that originate from $M_{1}$ and $M_{2}$, respectively (see Table 2). Typically, $E_{1}$ and $E_{2}$ will lie very close to the $M_{1}-X-M_{2}$ plane, but for generality we project $E_{1}$ and $E_{2}$ onto this plane. To determine the configuration of $M_{i}$ with respect to its external environment, the $\mathrm{M}_{i}-\mathrm{E}_{i}-\mathrm{E}_{j}$ angle $\theta_{i}$ is calculated. This is then compared to the analogous angle $\theta_{i}^{\prime}$ in the alternate isomer produced by BAI. Here we need to choose an arbitrary numeral criterion for comparing the angles; by testing many $\mathrm{B}-\mathrm{O}-\mathrm{B}$ complexed macrocycles including corroles (see Supplementary Information Section 8) we found that comparing $\theta_{i} / \theta_{i}^{\prime}$ values to 2/3 and $3 / 2$ appear to be a robust criterion. Accordingly, we define the descriptors as follows:

\section{$\theta_{i} / \theta_{i}^{\prime}<2 / 3: \quad M_{i}$ is assigned the stereodescriptor parvo (from Latin meaning "small”), \\ $2 / 3 \leq \theta_{i} / \theta_{i}^{\prime} \leq 3 / 2$ : no stereodescriptor applies, \\ $\theta_{i} / \theta_{i}^{\prime}>3 / 2: \quad M_{i}$ is assigned the stereodescriptor amplo (from Latin meaning "large”).}

Table 2 shows the application of this scheme to 2a-5a, also the BAI transition state $\mathbf{T S}_{\mathrm{ak}}^{R}$ connecting 2a with 3a and a defluorinated reaction intermediate, illustrating that this method leads to unique chemical names. When $\mathrm{M}_{1}$ and $\mathrm{M}_{2}$ are stereocentres, they are labelled either " $R$ " or " $S$ ", to which the right subscript labels " $a$ " for "amplo" and " $p$ " for "parvo" are added. The labels for each atom $\mathrm{M}_{i}$ in the name are ordered according to the standard numbering system of the surrounding macrocycle. Abbreviated names are also provided in the figure. All $\mathbf{b}$ isomers are named similar to the a isomers shown, with all $R$ and $S$ labels interchanged. In Supplementary Information Section 8, the names and angles are given for the 36 porphyrin and corrole structures and transition states mentioned in this work.

Compounds like 2-5 form two bonds from each of $\mathrm{M}_{1}$ and $\mathrm{M}_{2}$, to their environments, within a macroscopic ring system. Then (parvo) configurations present $\mathrm{M}$ atoms in-plane ("plano") to the environment whilst (amplo) configurations present them out-of-plane ("aplano"), connecting to the intuitive structural relationships highlighted in yellow in Figure 3.

Isolable akamptisomers arise from compression of the $(\mathrm{BF}) \mathrm{O}(\mathrm{BF})$ group by the porphyrin macrocycle. For akamptisomers to be isolable at room temperature, the BAI barrier must be sizeable. The intrinsic barrier to making $\mathrm{M}_{1}-\mathrm{X}-\mathrm{M}_{2}$ bonds linear can vary substantially ${ }^{66}$.

\section{Conclusions}


The polytope formalism of conformational isomerisation has been generalised to include internal rotations. This systematic analysis of simple systems containing four or fewer atoms established that one conformational isomerisation process, BAI, can be identified as a fundamental process that can create new isomers not achievable by other means. In particular, we have demonstrated that a hindered BAI at room temperature in singly bonded systems represents a form of conformational isomerism that we call akamptisomerism. $\beta, \beta^{\prime}$-quinoxalinoporphyrin complexes of (BF)O(BF) were synthesised and shown to produce isolable chiral akamptisomers, with asymmetric substitution of the porphyrin used to differentiate between isomers and enhance separation and characterisation. Although eight isomers may exist, we detected, isolated and characterized only the four transoid isomers, obtained in near-equal yield.

While the discovery of akamptisomers demands only the isolation of molecules related by conceptual BAI at a singly bonded atom, we also observed the synthesised compounds actually thermally interconverting this way. As the resulting akamptisomers and the BAI processes interconnecting them could not be named using existing IUPAC nomenclature, we have taken into consideration all possible products as well as their related transition states and reactions to develop a robust and informative naming convention for structures related by BAI.

This discovery of akamptisomerism parallels the discovery of atropisomerism in which unexpectedly large barriers to rotation allow rotamers about single bonds to be isolated rather than just rotamers involving double bonds. Here, BAI involving a single bonded atom is shown to involve unexpectedly large barriers, allowing isolable isomers not involving double bonding.

We expect that BAI will be widely observable in macrocyclic systems encircling bent $\mathrm{M}_{1}-\mathrm{X}-\mathrm{M}_{2}$ groups, binding $M_{1}$ at $E_{1}$ and $M_{2}$ at $E_{2}$ within the macrocycle. Many possibilities exist for $M_{1}, X$ and $M_{2}$ as well as for the macrocycle linkages $E_{1}-E_{2}$. While we have focused on macrocyclic ligands as providing a controlled external environment, the specific linkages in polymers and proteins are also naturally susceptible to BAI.

\section{Methods}

The full synthetic details for the reaction shown in Figure 3 are given in Supplementary Information Section 2. Briefly, $\mathrm{Et}_{2} \mathrm{O} \cdot \mathrm{BF}_{3} / \mathrm{NEt}_{3}$ was added to the free base quinoxalinoporphyrin in excess, the reaction being performed in $\mathrm{CH}_{2} \mathrm{Cl}_{2}$ solution at $50^{\circ} \mathrm{C}$. This inserted the two boron atoms in the macrocycle cavity, with the central $\mathrm{B}-\mathrm{O}-\mathrm{B}$ bonds formed by subsequent treatment with $1 \mathrm{M}$ aqueous $\mathrm{NaOH}$. This gave the racemates 2 (39\% yield) and 3 (40\% yield) as brilliant emerald-green products. As expected, the structures of racemates $\mathbf{2}$ and $\mathbf{3}$ are easily distinguishable by NMR owing to large ring current effects coming from the porphyrino and quinoxalino groups.

Purification of the four major reaction products, enantiomeric pairs $\mathbf{2} \mathbf{a} / \mathbf{2} \mathbf{b}$, and $\mathbf{3 a} \mathbf{a} \mathbf{3} \mathbf{b}$, was performed (Supplementary Information Section 2) by repeated chiral HPLC on a Jones Apex PK analytical (Jones Pirkle type 1-A) semi-prep column, eluent hexane:2-propanol 99:1, V:V. The products were investigated using ${ }^{1} \mathrm{H}$ NMR, ${ }^{19} \mathrm{~F}$ NMR, absorption, circular dichroism (CD), and magnetic circular dichroism (MCD) spectroscopies ${ }^{67}$ in solution and in solvent glasses ${ }^{68}$, with the absorption and MCD data analysed by analytic data inversion. ${ }^{69}$ This is described in detail in Supplementary Information Sections 3-6. Briefly, distinguishability of isomers is envisaged using CD spectroscopy as porphyrin to quinoxaline charge-transfer bands ${ }^{70}$ will be generated that can sense both the innerring environment and the presence of distant quinoxalino substituents. These transitions mix with the intense Soret bands to give significant changes to CD spectra as expected. Product chirality is tentatively assigned in Supplementary Information Section 7, considering both these results and the 
observed thermal isomerisation pathways, based on DFT calculations. In these, geometric structures were obtained using the B3LYP functional ${ }^{71}$, with spectra simulated using this plus the CAMB3LYP $^{72-74}$ and WB97XD $^{75}$ functionals as well as the configuration-interaction-singles (CIS) method $^{76}$ by Gaussian09 ${ }^{77}$, (Supplementary Information Section 6). Grimme’s empirical dispersion correction $^{78}$ "D3" was also used, as was the polarised continuum model for simulating solvent effects $^{79}$ including dispersion ${ }^{80}$; gauge invariant basis sets were used for NMR calculations ${ }^{81}$. The general calculation principles, previously developed for chlorophylls and porphyrins ${ }^{82,83}$, were utilised. Some coupled-cluster singles and doubles calculations with perturbative correction for triples (CCSD(T)) were also performed by MOLPRO ${ }^{84}$ using the cc-pVDZ and cc-pVTZ ${ }^{85}$ basis sets.

\section{Data Availability}

All raw experimental data discussed in this work is presented in Supplementary Information. Also included in Supporting Data are the optimised coordinates for all discussed compounds.

\section{Acknowledgments}

We thank the University of Sydney for a Gritton Scholarship, the Australian Research Council (grants DP0666378, DP0773847 and DP150103137), the National Natural Science Foundation of China (NSFC; Grant No. 11674212), and the Shanghai High-End Foreign Experts Grant for funding this research, as well as National Computational Infrastructure (NCI, d63) and INTERSECT (r88) for the provision of computing resources. A special thanks to Graham Price for his help with the Latin terms. This work is dedicated to the stereochemistry pioneer Kurt Mislow 1923-2017.

\section{Author contributions}

P.J.C., J.R.R., and M.J.C. conceived and designed the overall project and wrote the manuscript. I.M.B. was primarily responsible for synthesis (with help from P.J.C. and M.J.C). I.J.L. designed and performed the NMR studies. P.J.C. performed the chiral resolutions, UV/Vis, CD and MCD spectroscopies, along with and designed by E.K.. P.J.C performed all structural optimisations and NMR calculations, whilst Z.-L.C. and R.K. designed and performed the UV/VIS spectral simulations with, in particular, R.K. focusing on the difficult question of accurate predictions of chirality. P.J.C. developed the application of the polytope formalism and the nomenclature study. The videos were prepared by P.J.C.

\section{References}

1 McNaught, A. D. \& Wilkinson, A. IUPAC Compendium of Chemical Terminology, 2nd ed. (the "Gold Book"). (Blackwell, Oxford, 1997).

2 Testa, B., Caldwell, J. \& Kisakürek, M. V. Organic stereochemistry: Guiding principles and bio-medicinal relevance: A general introduction to the series. Helv. Chim. Acta 96, 1-3 (2013).

3 Testa, B., Vistoli, G. \& Pedretti, A. Organic stereochemistry. Part 1. Symmetry elements and operations, classification of stereoisomers. Helv. Chim. Acta 96, 4-30 (2013).

4 Testa, B. Organic stereochemistry. Part 2: Stereoisomerism resulting from one or several stereogenic centers. Helv. Chim. Acta 96, 159-188 (2013).

5 Testa, B. Organic stereochemistry. Part 3: Other stereogenic elements: Axes of chirality, planes of chirality, helicity, and (E,Z)-diastereoisomerism. Helv. Chim. Acta 96, 351-374 (2013).

6 Testa, B., Vistoli, G. \& Pedretti, A. Organic stereochemistry. Part 4: Isomerisms about single bonds and in cyclic systems. Helv. Chim. Acta 96, 564-623 (2013). 
Testa, B., Vistoli, G., Pedretti, A. \& Caldwell, J. Organic stereochemistry. Part 5: Stereoselectivity in molecular and clinical pharmacology. Helv. Chim. Acta 96, 747-798 (2013).

8 Vistoli, G., Testa, B. \& Pedretti, A. Organic stereochemistry. Part 6: The conformation factor in molecular pharmacology. Helv. Chim. Acta 96, 1005-1031 (2013).

9 Testa, B. Organic stereochemistry. Part 7: The concept of substrate stereoselectivity in biochemistry and xenobiotic metabolism. Helv. Chim. Acta 96, 1203-1234 (2013).

Testa, B. Organic stereochemistry. Part 8: Prostereoisomerism and the concept of product stereoselectivity in biochemistry and xenobiotic metabolism. Helv. Chim. Acta 96, 14091451 (2013).

11 King, H. The possibility of a new instance of optical activity without an asymmetric carbon atom. Proc. Chem. Soc. London 30, 249-251 (1914).

Cain, J. C. \& Micklethwait, F. M. G. Studies in the diphenyl series. Part VI. The configuration of diphenyl and its derivatives. J. Chem. Soc. Trans. 105, 1437-1441 (1914).

Christie, G. H. \& Kenner, J. The molecular configurations of polynuclear aromatic compounds. Part I. The resolution of $\gamma$-6:6'-dinitro- and $4: 6: 4^{\prime}: 6^{\prime}$-tetranitro-diphenic acids into optically active components J. Chem. Soc. Trans. 121, 614-620 (1922). Kuhn, R. Molekulare asymmetrie. 803-824 (Franz-Deutike, Leipzig-Wien, 1933). Horner, L. et al. Phosphororganische verbindungen optisch aktive tertiäre phosphine aus optisch aktiven quartären phosphoniumsalzen. Tetrahedron Lett. 2, 161-166 (1961).

Brois, S. J. Aziridines. XII. Isolation of a stable nitrogen pyramid. J. Am. Chem. Soc. 90, 508-509 (1968).

Muetterties, E. L. Topological representation of stereoisomerism. I. Polytopal rearrangements. J. Am. Chem. Soc. 91, 1636-1643 (1969).

18 Muetterties, E. L. \& Storr, A. T. Topological analysis of polytopal rearrangements. Sufficient conditions for closure. J. Am. Chem. Soc. 91, 3098-3099 (1969).

19 Muetterties, E. L. Topological representation of stereoisomerism. II. The five-atom family. $J$. Am. Chem. Soc. 91, 4115-4122 (1969).

20 Meakin, P. et al. Structure and stereochemical nonrigidity of six-coordinate complexes. $J$. Am. Chem. Soc. 92, 3482-3484 (1970).

21 Muetterties, E. L., Wiersema, R. J. \& Hawthorne, M. F. Detection of polytopal isomers in the solution state. I. Eight-atom family. J. Am. Chem. Soc. 95, 7520-7522 (1973).

Muetterties, E. L. \& Guggenberger, L. J. Idealized polytopal forms. Description of real molecules referenced to idealized polygons or polyhedra in geometric reaction path form. $J$. Am. Chem. Soc. 96, 1748-1756 (1974).

23 Muetterties, E. L. Polytopal form and isomerism. Tetrahedron 30, 1595-1604 (1974).

24 Guggenberger, L. J. \& Muetterties, E. L. Reaction path analysis. 2. The nine-atom family. J. Am. Chem. Soc. 98, 7221-7225 (1976).

25 Hoffmann, R., Beier, B. F., Muetterties, E. L. \& Rossi, A. R. Seven-coordination. A molecular orbital exploration of structure, stereochemistry, and reaction dynamics. Inorg. Chem. 16, 511-522 (1977).

26 Debolt, L. C. \& Mark, J. E. Effects of bond-angle inversion on the statistical properties of poly(dimethylsiloxane). J. Polym. Sci., Part B: Polym. Phys. 26, 989-995 (1988).

27 Kalinowski, H.-O. \& Kessler, H. in Topics in steroechemistry Vol. 7 N.L. Allinger \& E.l. Eliel (eds.) 295-384 (Wiley Interscience, new York, 1973).

28 Kessler, H. Thermal isomerization about double bonds. Rotation and inversion. Tetrahedron 30, 1861-1870 (1974).

29 Gozlan, H., Michelot, R., Riche, C. \& Rips, R. Amide-oximes: determination des configurations et etude du mecanisme de l'isomerisation Z-E. Tetrahedron 33, 2535-2542 (1977).

30 Bakhmutov, V. I. et al. A dynamic NMR study of Z, E-isomerization in solutions of indolylsubstituted $\alpha$-nitroacrylates. Organic Magnetic Resonance 11, 308-312 (1978). 
Appenroth, K., Reichenbächer, M. \& Paetzold, R. Thermochromism and photochromism of aryl substituted acyclic azines1 1 4. Communication; 3. Communication, see Ref. 4. Uncatalised and acid-catalised thermal isomerisation. Tetrahedron 37, 569-573 (1981).

Kirste, K. \& Rademacher, P. Rotation and inversion in nitrosamines. J. Mol. Struct. 73, 171180 (1981).

33 Sammes, M. P. The effect of salt formation on structure and charge distribution in imines. Part 4. Energy barriers to isomerisation about the C-N bond in 2,6-dimethyl-4aryliminopyrans and their salts: solvent and substituent effects, and evidence for isomerisation mechanisms. J. Chem. Soc., Perkin Trans. 2, 1501-1507 (1981).

34 Kawada, Y. \& Iwamura, H. Bis(4-chloro-1-triptycyl) ether. Separation of a pair of phase isomers of labeled bevel gears. J. Am. Chem. Soc. 103, 958-960 (1981).

Gustav, K., Vettermann, S. \& Birner, P. Quantum chemical studies on colour and stereodynamics of acyclic azines. Part IX. The coupled $\mathrm{NN}$ rotation/ $\mathrm{N}$ inversion mechanism for the thermal (E,Z) isomerization of benzaldazine. THEOCHEM 88, 249-253 (1982).

36 Kawada, Y. \& Iwamura, H. Correlated rotation in bis(9-triptycyl)methanes and bis(9triptycyl) ethers. Separation and interconversion of the phase isomers of labeled bevel gears. J. Am. Chem. Soc. 105, 1449-1459 (1983).

37 Cunningham, I. D. \& Hegarty, A. F. Acid, base, and uncatalysed isomerisation of Z- to Eamidine. A mechanistic study. J. Chem. Soc., Perkin Trans. 2, 537-541 (1986).

38 Frenna, V., Buscemi, S., Spinelli, D. \& Consiglio, G. A kinetic study on the base-catalysed $\mathrm{e} \rightarrow \mathrm{z}$ isomerization of some arylhydrazones of 3-benzoyl-5-phenyl-1,2,4-oxadiazole: effect of the substituents in the arylhydrazone moiety. J. Chem. Soc., Perkin Trans. 2, 215-221 (1990). Gáplovský, A., Donovalová, J., Lacová, M., Mračnová, R. \& El-Shaaer, H. M. The photochemical behaviour of 6-X-4H-3(bicyclo[2.2.1]-5-heptene-2,3dicarboximidoiminomethyl)-4-chromones. Photochromism and thermochromism. $J$. Photochem. Photobiol., A 136, 61-65 (2000).

40 Sung, K. N-substituent effects on the stability of ketenimines. J. Chem. Soc., Perkin Trans. 2, 847-852 (2000).

41 Klika, K. D. et al. Configuration and E/Z interconversion mechanism of O(S)-allyl-S(O)methyl- N-(acridin-9-yl)-iminothiocarbonate. Magn. Reson. Chem. 43, 380-388 (2005).

42 Pirozhenko, V. V., Rozhenko, A. B., Avdeenko, A. P., Konovalova, S. A. \& Santalova, A. A. $\mathrm{Z}$, E-Isomerization mechanism for $\mathrm{N}$-arylthio-1,4-benzoquinonimines: DNMR and DFT investigations. Magn. Reson. Chem. 46, 811-817 (2008).

43 Landge, S. M. et al. Isomerization mechanism in hydrazone-based rotary switches: Lateral shift, rotation, or tautomerization? J. Am. Chem. Soc. 133, 9812-9823 (2011).

44 Luo, Y. et al. Cis-trans isomerisation of substituted aromatic imines: A comparative experimental and theoretical study. ChemPhysChem 12, 2311-2321 (2011).

45 Greb, L., Eichhöfer, A. \& Lehn, J. M. Synthetic Molecular Motors: Thermal N Inversion and Directional Photoinduced $\mathrm{C}=\mathrm{N}$ Bond Rotation of Camphorquinone Imines. Angew. Chem. Int. Ed. 54, 14345-14348 (2015).

46 Belcher, W. J., Boyd, P. D. W., Brothers, P. J., Liddell, M. J. \& Rickard, C. E. F. J. Am. Chem. Soc. 116, 8416-8417 (1994).

47 Belcher, W. J., Breede, M., Brothers, P. J. \& Rickard, C. E. F. The porphyrin as a binucleating ligand: preparation and crystal structure of a porphyrin complex containing a coordinated B2O2 ring. Angew. Chem. Int. Ed. 37, 1112-1114 (1998).

48 Brothers, P. J. Organometallic chemistry of main group porphyrin complexes. Adv. Organomet. Chem. 48, 289-295 (2001).

49 Brothers, P. J. Recent developments in the coordination chemistry of porphyrin complexes containing non-metallic and semi-metallic elements. J. Porphyrins Phthalocyanines 6, 259267 (2002).

50 Köhler, T. et al. Octaethylporphyrin and expanded porphyrin complexes containing coordinated $\mathrm{BF}_{2}$ groups. Chem. Commun., 1060-1061 (2004). 
Albrett, A. M., Boyd, P. D. W., Clark, G. R., Gonzalez, E. \& Brothers, P. J. Reductive coupling and protonation leading to diboron corroles with a B-H-B bridge. Dalton Trans. 39, 4032-4034 (2010).

Belcher, W. J. et al. Porphyrin complexes containing coordinated BOB groups: synthesis, chemical reactivity and the structure of [BOB(tpClpp) $]^{2+}$. Dalton Trans., 1602-1614 (2008). Brothers, P. J. Boron complexes of porphyrins and related polypyrrole ligands: unexpected chemistry for both boron and the porphyrin. Chem. Commun., 2090-2102 (2008).

Albrett, A. PhD Thesis: Synthesis of Boron Corrole Complexes. (The University of Auckland, Auckland, 2009).

Brothers, P. J. Boron Complexes of Pyrrolyl Ligands. Inorg. Chem. 50, 12374-12386 (2011). Hydrolytic Reactivity. Inorg. Chem. 53, 5486-5493 (2014).

B3LYP-D3/6-31G* calculations indicate the unobserved BAI isomer is less stable by $\Delta G=$ $30 \mathrm{~kJ} \mathrm{~mol}^{-1}$, see Supplementary Information Section S8.

Weiss, A., Hodgson, M. C., Boyd, P. D. W., Siebert, W. \& Brothers, P. J. Diboryl and diboranyl porphyrin complexes: synthesis, structural motifs, and redox chemistry: diborenyl porphyrin or diboranyl isophlorin? Chem. - Eur. J. 13, 5982-5993 (2007).

Albrett, A. M. et al. Corrole as a binucleating ligand: preparation, molecular structure and density functional theory study of diboron corroles. J. Am. Chem. Soc. 130, 2888-2889 (2008).

Albrett, A. M., Conradie, J., Ghosh, A. \& Brothers, P. J. DFT survey of monoboron and diboron corroles: regio- and stereochemical preferences for a constrained, low-symmetry macrocycle. Dalton Trans., 4464-4473 (2008).

61 Crossley, M. J., Sintic, P. J., Walton, R. \& Reimers, J. R. Synthesis and physical properties of biquinoxalinyl bridged bis-porphyrins: models for aspects of Photosynthetic Reaction Centres. Org. Biomol. Chem. 1, 2777-2787 (2003).

Kadish, K. M. et al. Quinoxalino[2,3-b']porphyrins Behave as p-Expanded Porphyrins upon One-Electron Reduction: Broad Control of the Degree of Delocalization through Substitution at the Macrocycle Periphery. J. Phys. Chem. B 111, 8762-8774 (2007).

Sintic, P. J. et al. Control of the site and potential of reduction and oxidation processes in piexpanded quinoxalinoporphyrins. Phys. Chem. Chem. Phys. 10, 268-280 (2008).

Hartshorn, R. The red book - Nomenclature of inorganic chemistry. IUPAC Recommendations 2005. Chem. Int. 29, 14-16 (2007).

65 Dixon, H. B. F. et al. Nomenclature of tetrapyrroles. Pure Appl. Chem. 59, 779-832 (1987).

66 Goerigk, L. \& Sharma, R. The INV24 test set: how well do quantum-chemical methods describe inversion and racemization barriers? Can. J. Chem. 94, 1133-1143 (2016).

Krausz, E. Selective and differential optical spectroscopies in photosynthesis. Photosynth. Res. 116, 411-426 (2013).

68 Scott, D. R. S. \& Allison, J. B. Solvent glasses for low temperature spectroscopic studies. J. Phys. Chem. 66, 561-562 (1962).

69 Reimers, J. R. \& Krausz, E. An analytical data inversion method for Magnetic Circular Dichroism spectra dominated by the "B-term". Phys. Chem. Chem. Phys. 16, 2315-2322 (2014).

70 Sendt, K. et al. Switchable electronic coupling in oligoporphyrin molecular wires examined through the measurement and assignment of electronic absorption spectra. J. Am. Chem. Soc. 124, 9299-9309 (2002).

71 Becke, A. D. Density-functional thermochemistry. III. The role of exact exchange. J. Chem. Phys. 98, 5648-5652 (1993).

72 Yanai, T., Tew, D. P. \& Handy, N. C. A new hybrid exchange-correlation functional using the Coulomb-attenuating method (CAM-B3LYP). Chem. Phys. Lett. 393, $51-57$ (2004).

Kobayashi, R. \& Amos, R. D. The application of CAM-B3LYP to the charge-transfer band problem of the zincbacteriochlorin-bacteriochlorin complex. Chem. Phys. Lett. 420, 106-109 (2006). 
Cai, Z.-L., Crossley, M. J., Reimers, J. R., Kobayashi, R. \& Amos, R. D. Density-functional theory for charge-transfer: the nature of the N-bands of porphyrins and chlorophylls revealed through CAM-B3LYP, CASPT2, and SAC-CI calculations. J. Phys. Chem. B 110, 1562415632 (2006).

75 Chai, J.-D. \& Head-Gordon, M. Long-range corrected hybrid density functionals with damped atom-atom dispersion corrections. Phys. Chem. Chem. Phys. 10, 6615-6620 (2008).

76 Foresman, J. B., Head-Gordon, M., Pople, J. A. \& Frisch, M. J. towards a systematic molecular orbital theory for excited states. J. Phys. Chem. 96, 135 (1992).

77 Frisch, M. J. et al. Gaussian 09, Revision D.01. (Gaussian, Inc., Pittsburgh PA, 2009).

78 Goerigk, L. \& Grimme, S. A thorough benchmark of density functional methods for general main group thermochemistry, kinetics, and noncovalent interactions. Phys. Chem. Chem. Phys. 13, 6670-6688 (2011).

79 Tomasi, J., Mennucci, B. \& Cammi, R. Quantum mechanical continuum solvation models. Chem. Rev. 105, 2999-3093 (2005).

80 Floris, F. M., Tomasi, J. \& Pascual Ahuir, J. L. Dispersion and Repulsion Contributions to the Solvation Energy: Refinements to a Simple Computational Model in the Continuum Approximation. J. Computat. Chem. 12, 784-791 (1991).

81 Wolinski, K., Hinton, J. F. \& Pulay, P. Efficient implementation of the gauge-independent atomic orbital method for NMR chemical shift calculations. J. Am. Chem. Soc. 112, 82518260 (1990).

82 Kobayashi, R. \& Reimers, J. R. Free energies for the coordination of ligands to the magnesium of chlorophyll-a in solvents. Mol. Phys. 16, 928-932 (2015).

83 Reimers, J. R. et al. Assignment of the Q-Bands of the Chlorophylls: Coherence Loss via Qx - Qy Mixing. Sci. Rep. 3, 2761 (2013).

84 Werner, H.-J. et al. MOLPRO, version 2015.1, a package of ab initio programs. (University of Birmingham, Birmingham, 2015).

85 Dunning, T. H., Jr. Gaussian basis sets for use in correlated molecular calculations I. The atoms boron through neon and hydrogen. J. Chem. Phys. 90, 1007 (1989). 


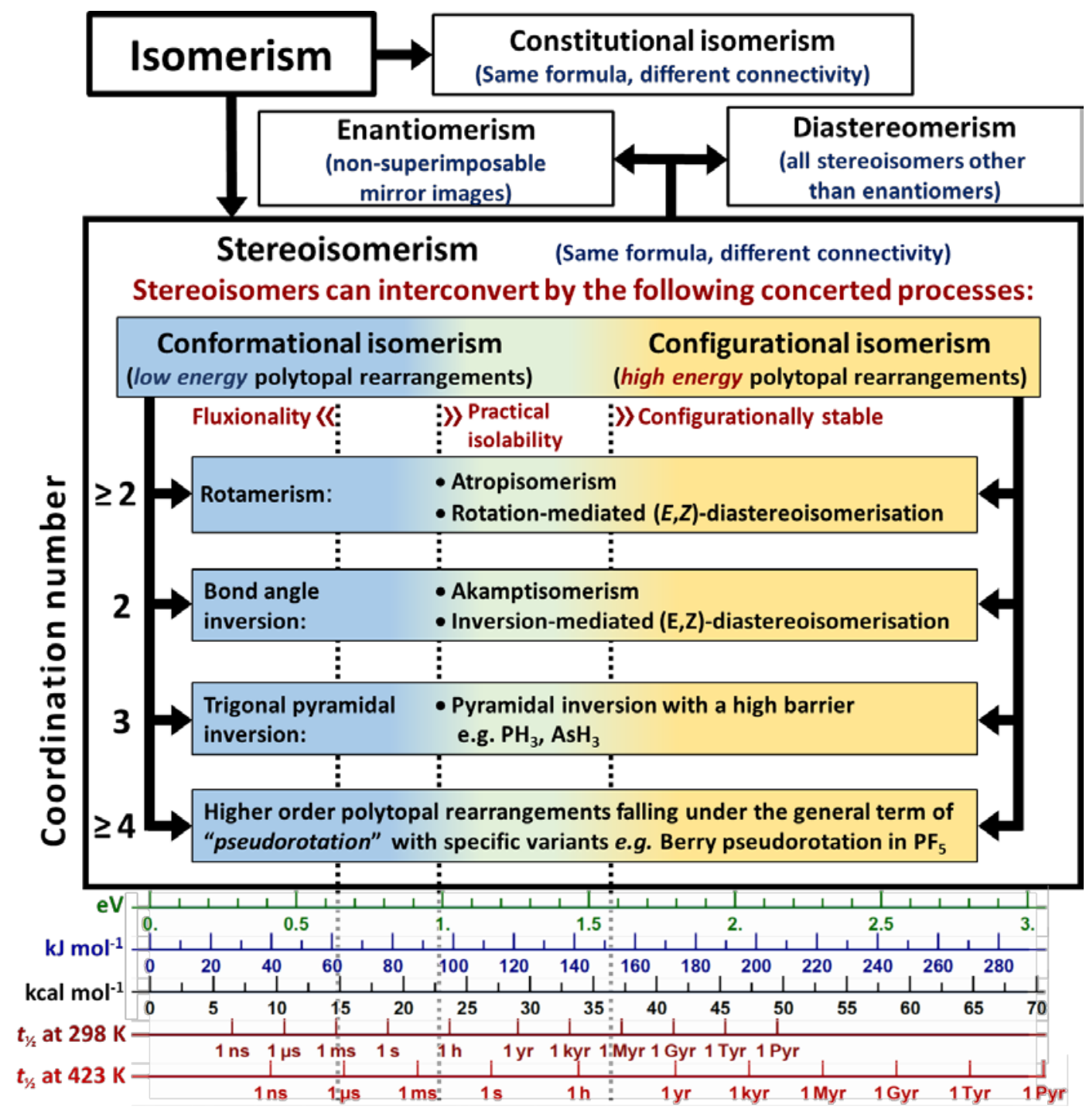

Figure 1 | Isomerism Hierarchy. IUPAC classifications of isomerism and isomerisation types with emphasis on stereoisomerism, extended to include akamptisomerisation associated with bond angle inversion within the overall framework of the polytope formalism specifying feasible reaction processes. The different processes encompass a range of phenomena characterised by the energetic barriers involved. The horizontal extent and shading of the rectangles within the stereoisomerism box indicate typical energy ranges for the mechanisms they enclose, as read with reference to the scales for the energy barriers and the temperature-dependent first order half-life of the corresponding species shown at the bottom of the figure. The positions of the dotted vertical lines indicate suggested property delineations, with "fluxionality" reflecting an interconversion between stereoisomers that is too rapid to observe, "practical isolability" representing species stable for 1000 s at $298 \mathrm{~K}$, and “configurationally stable” stable for 24 hours at $423 \mathrm{~K}$. 


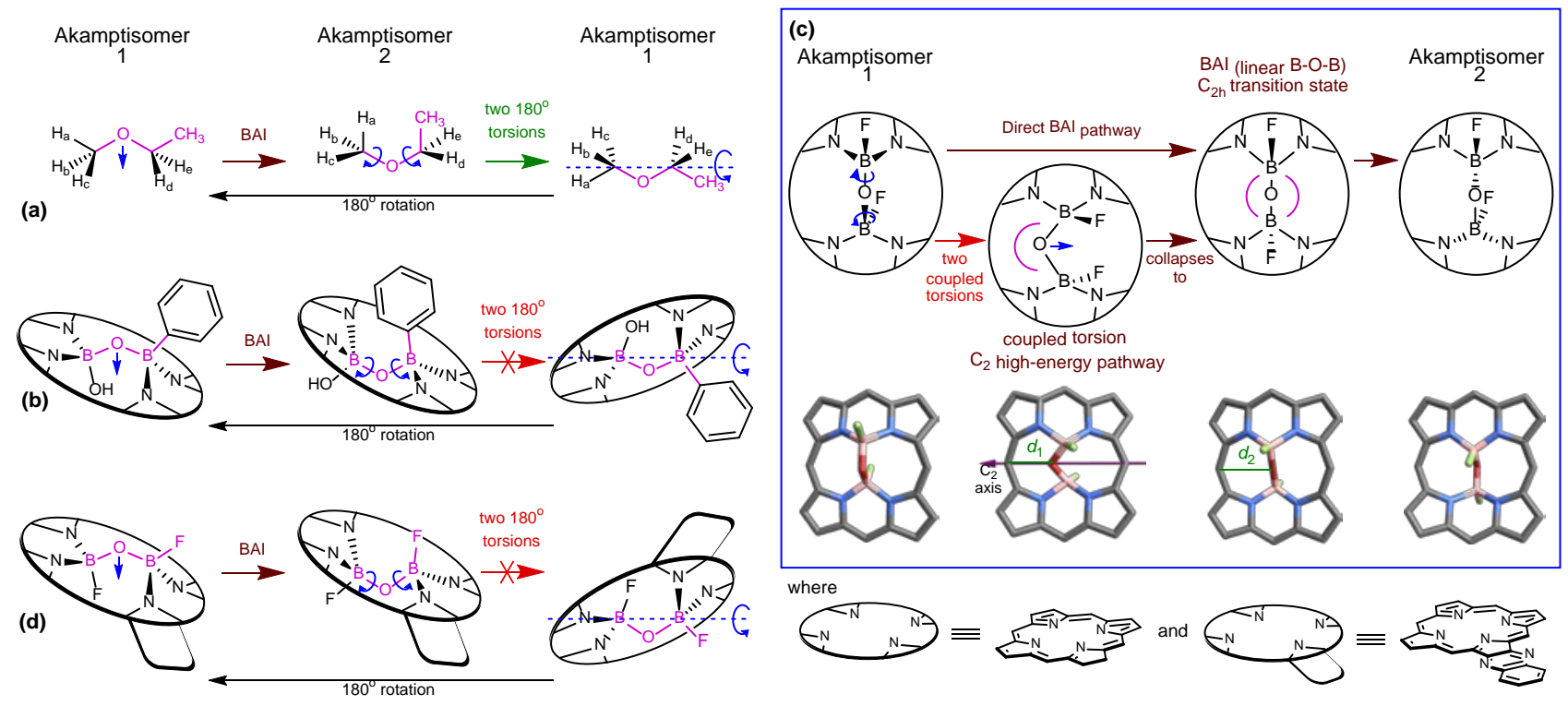

Figure 2 | BAI processes within single bonds. (a) BAI can conceivably occur in many molecules but coupled torsional motions undo the reaction at minimal energy cost, with two $180^{\circ}$ torsional motions followed by $180^{\circ}$ rotation demonstrating the effect most simply. (b) Side view of $\mathrm{B}-\mathrm{O}-\mathrm{B}$ showing that embedding the atoms of interest with a macrocycle couples and sterically hinders such torsional motions, producing distinct akamptisomers. (c) Top view of macrocycle featuring an alternative double torsion involving only $90^{\circ}$ motions could also undo BAI, but owing to compression from the macrocycle, double-torsion pathways involve strong steric repulsions (shown B3LYP/6-31G* optimised O-C non-bonded distance $d_{1}=2.27 \AA$ ) are all of higher energy than the BAI transition state $\left(\mathrm{C}_{2 \mathrm{~h}}\right.$ optimised $\mathrm{O}-\mathrm{C}$ non-bonded distance $d_{2}=2.94 \AA$ ) and so do not undo the process. (d) Asymmetric substitution of the macrocycle allows BAI to produce a distinct akamptisomer that also cannot revert through coupled torsional motions. Views (a), (b), and (d) show the fundamental processes with respect to the magenta-coloured $\mathrm{B}-\mathrm{O}-\mathrm{B}$ (or $\mathrm{C}-\mathrm{O}-\mathrm{C}$ ) atoms placed at characteristic positions and hence involve large motions of the surrounding atoms, whereas in (c) the view is with respect to the macrocycle, showing the $\mathrm{O}$ atom moving through it. Atomic colours: white- $\mathrm{H}$, peach- $\mathrm{B}$, grey- $\mathrm{C}$, blue- $\mathrm{N}$, red- $\mathrm{O}$, green- $\mathrm{F}$. 
(a)

(b)

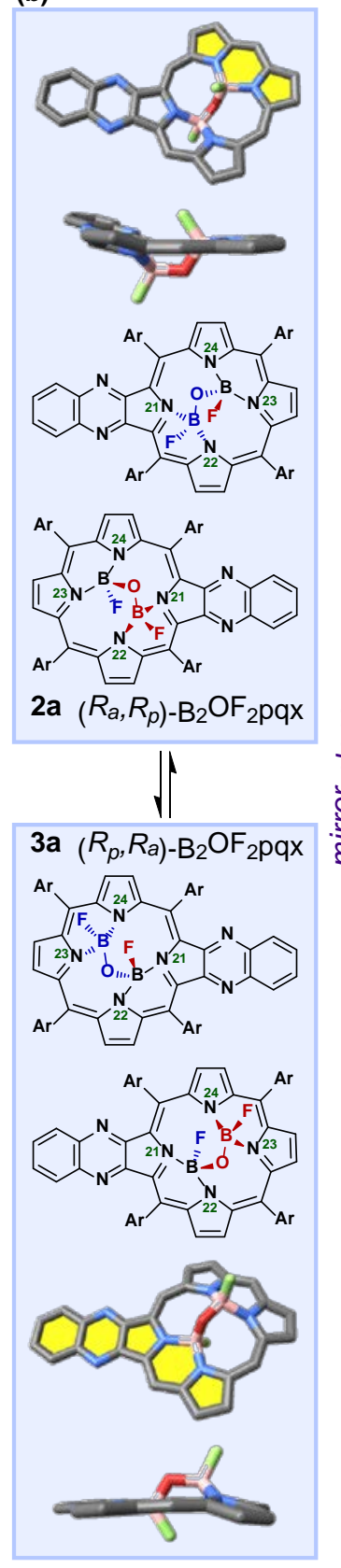

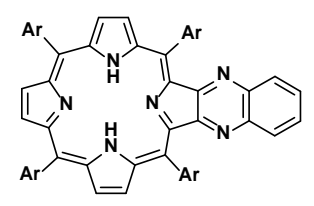

$1 \mathrm{Ar}=3,5$-di-tert-butylphenyl

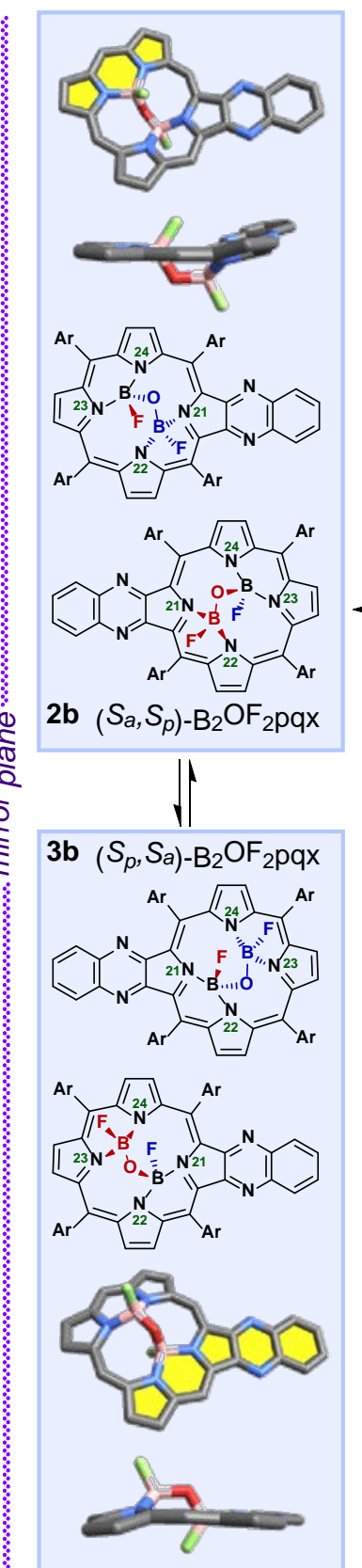

i) $\mathrm{BF}_{3} \cdot \mathrm{Et}_{2} \mathrm{O}$,

$\mathrm{CH}_{2} \mathrm{Cl}_{2} / \mathrm{NEt}_{3} / 50^{\circ} \mathrm{C}$

ii) $1 \mathrm{M} \mathrm{NaOH}(\mathrm{aq})$
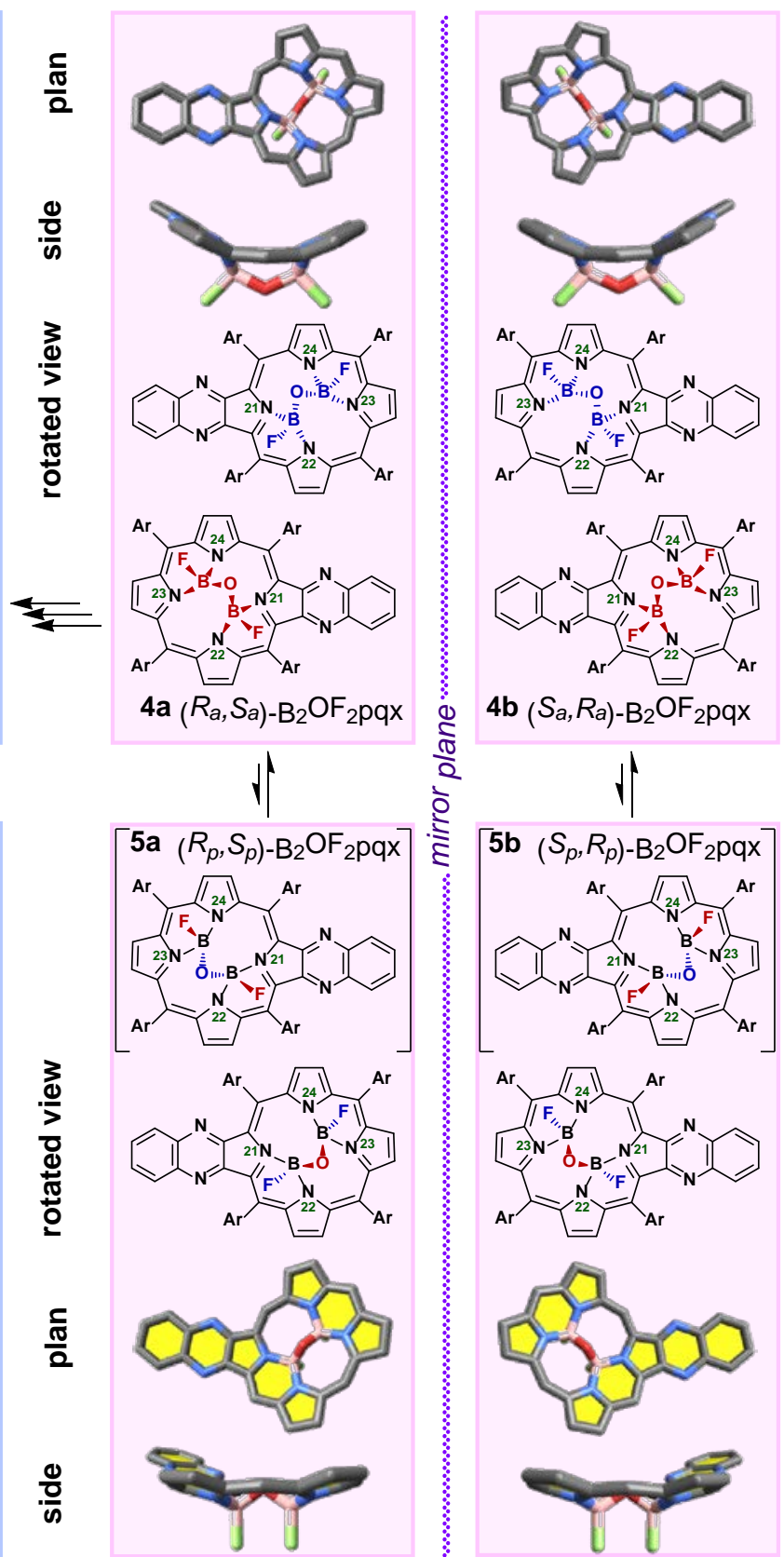

Figure 3 | Synthesis, structures and nomenclature of the eight possible stereoisomers produced by reaction of quinoxalinoporphyrin 1. a: Structure of $\mathbf{1}$ and reaction conditions. b: Only 2a, 2b, $\mathbf{3 a}$, and $\mathbf{3 b}$ (blue boxes) are actually isolated from this synthesis, with DFT suggesting $\mathbf{4 a}$ and $\mathbf{4 b}$ are much less stable kinetically and thermodynamically whilst $\mathbf{5 a}$ and $\mathbf{5 b}$ are unstable (pink boxes). Two equivalent representations of each structure are given, related by $180^{\circ}$ horizontal rotation. Enantiomers, for example $\mathbf{2 a}$ and $\mathbf{2} \mathbf{b}$, are related by vertical reflection, as indicated, whilst akamptisomers, such as 2a and 3a, are related by BAI, pushing the $\mathrm{O}$ through the effective plane of the macrocycle. All indicated stereochemistry is relative to the porphyrin ligand. Atoms and bonds marked in red are above the pseudoplane of the porphyrin whilst those in blue are below it. Hydrogen atoms and the aryl side chains are omitted for clarity, with rings co-planar to a chelated parvo boron atom shaded yellow. Atomic colours: white- H, peach- B, grey- C, blue- N, red- O, green- F. 


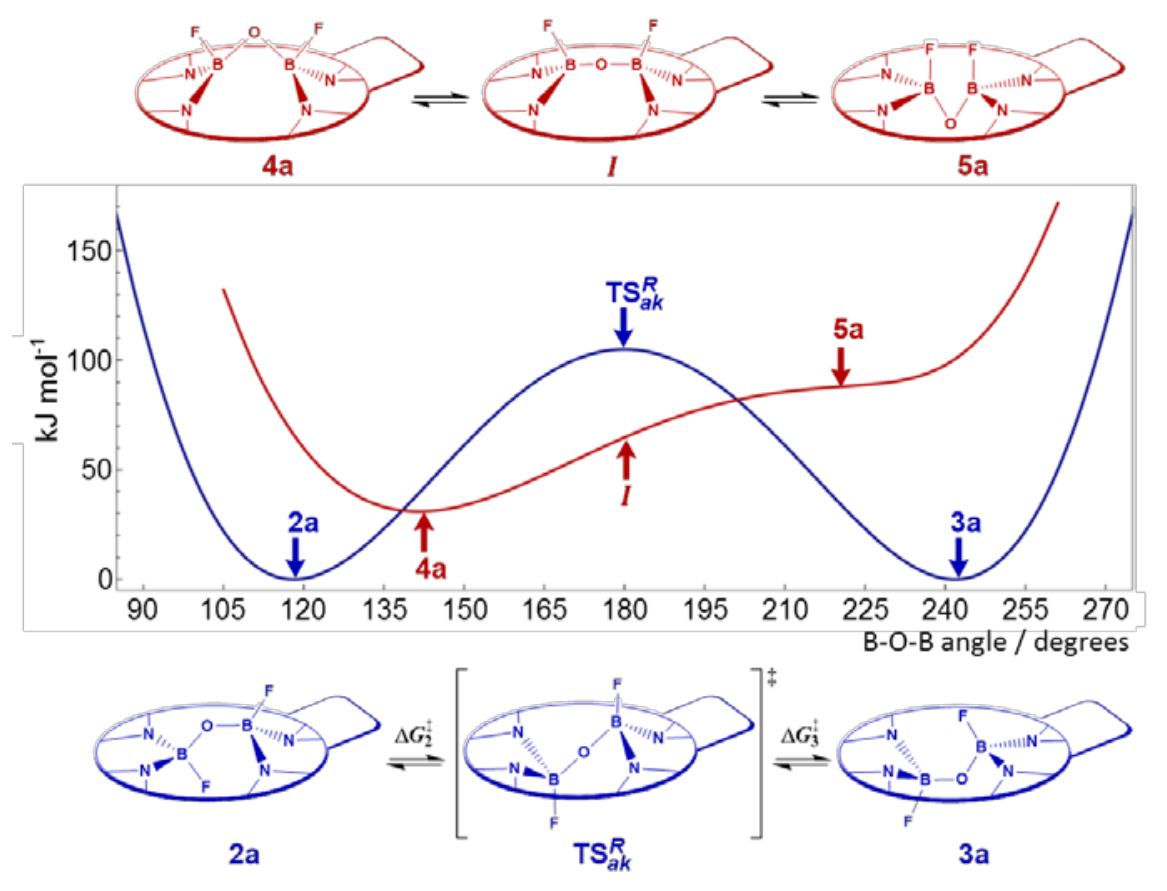

Figure 4 | Energetics of akamptisomerisation. DFT calculations show the metastable decay of the higher-energy cisoid compound 5a to the lower energy specie 4a through an intermediate I (shown in red, corresponding to the top structures) and the equilibrium over a BAI transition state $\mathbf{T S}_{\mathbf{a k}}^{R}$ between transoid compounds 2a and 3a (shown in blue, bottom structures). The meso 3,5-di-tertbutylphenyl groups and hydrogens are omitted for clarity. 


\section{TOC graphic}

colour code: carbon- grey, hydrogen- white, boron- peach, nitrogen- blue, oxygen- red, fluorinegreen

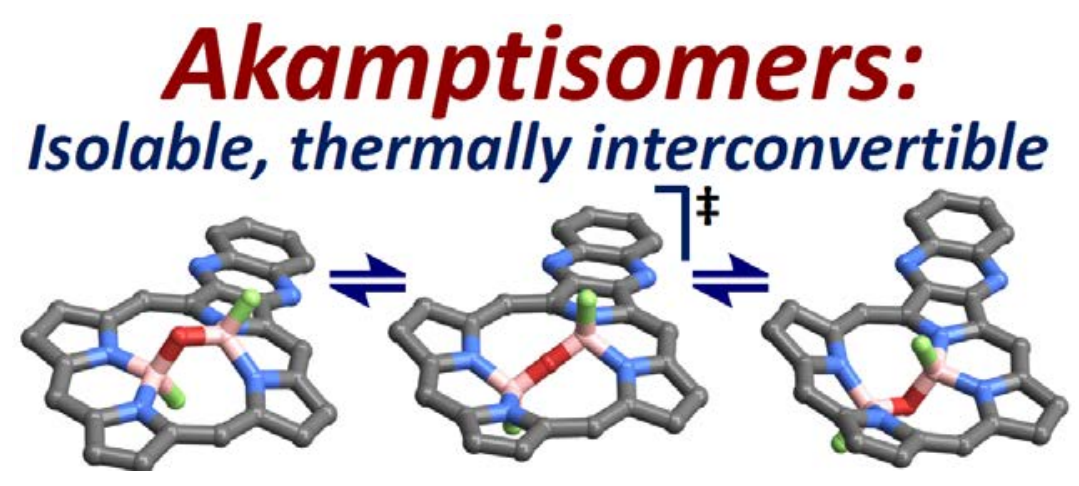


Table 1 | Observed and B3LYP/6-31G+(d)-calculated reaction activation free energies in $\mathrm{kJ} \mathrm{mol}^{-1}$, for various types of isomerisation mechanisms and reaction conditions. ${ }^{a}$

\begin{tabular}{|c|c|c|c|c|c|c|c|c|}
\hline \multirow[t]{2}{*}{ Environment } & \multirow[t]{2}{*}{ Process } & \multicolumn{2}{|c|}{ Akamptisomerisation } & \multicolumn{2}{|c|}{ Enantiomerisation } & \multicolumn{3}{|c|}{ cisoid relaxation } \\
\hline & & $\begin{array}{l}2 a \rightleftharpoons 3 a \\
3 b \rightleftharpoons 2 b\end{array}$ & $\begin{array}{l}3 a \rightleftharpoons 2 a \\
2 b \rightleftharpoons 3 b\end{array}$ & $2 a \rightleftharpoons 2 b$ & $3 a \rightleftharpoons 3 b$ & $\begin{array}{l}4 a \rightarrow 2 a \\
4 a \rightarrow 3 b \\
4 b \rightarrow 2 b \\
4 b \rightarrow 3 a\end{array}$ & $\begin{array}{l}4 a \rightarrow 2 a \\
4 b \rightarrow 3 b\end{array}$ & $\begin{array}{l}4 a \rightarrow 2 b \\
4 b \rightarrow 3 b\end{array}$ \\
\hline \multirow{4}{*}{$\mathrm{CH}_{2} \mathrm{Cl}_{2}$ solution } & $\mathrm{B}-\mathrm{O}-\mathrm{B} \mathrm{BAI}$ & 108 & 108 & - & - & - & - & - \\
\hline & $(\mathrm{BF})-\mathrm{O}-(\mathrm{BF})$ rotation $^{b}$ & 225 & 225 & 251 & 247 & - & - & - \\
\hline & $\mathrm{F}^{-}$loss & 284 & 285 & 220 & 220 & - & - & - \\
\hline & Observed & $104 \pm 2$ & $104 \pm 2$ & & & & & \\
\hline \multirow{4}{*}{$\begin{array}{l}\text { reaction } \\
\text { conditions }\end{array}$} & $\mathrm{HNEt}_{3}{ }^{+}$catalysed F${ }^{-}$loss & 180 & 180 & 116 & 119 & - & - & - \\
\hline & $\begin{array}{c}\mathrm{BF}_{3} \cdot \mathrm{NEt}_{3} \text { catalysed } \mathrm{F}^{-} \\
\text {loss }\end{array}$ & 180 & 180 & 106 & 109 & - & - & - \\
\hline & Uncatalysed $\mathrm{F}^{-} \mathrm{S}_{\mathrm{N} 2}$ & - & - & - & - & 82 & - & - \\
\hline & $\begin{array}{c}\mathrm{F}^{-} \text {loss, counterfacial } \\
\text { addition }\end{array}$ & - & - & - & - & - & 143 & 149 \\
\hline
\end{tabular}

${ }^{a}$ see Supplementary Information Section 6 for details, including pathways and considered alternatives. ${ }^{b}$ Strepsisomerisation - See . Supplementary Information Section 8c. 
Table 2 | Naming convention for atoms involved in BAI processes, their application to 2a-5a, the BAI transition structure linking $\mathbf{2 a}$ and 3a, and a defluorinated reaction intermediate, listing full systematic names plus convenient abbreviations. The $\mathrm{B}-\mathrm{O}-\mathrm{B}$ plane is highlighted, and the meso 3,5-di-tert-butylphenyl groups and hydrogens are omitted for clarity.

Schematics

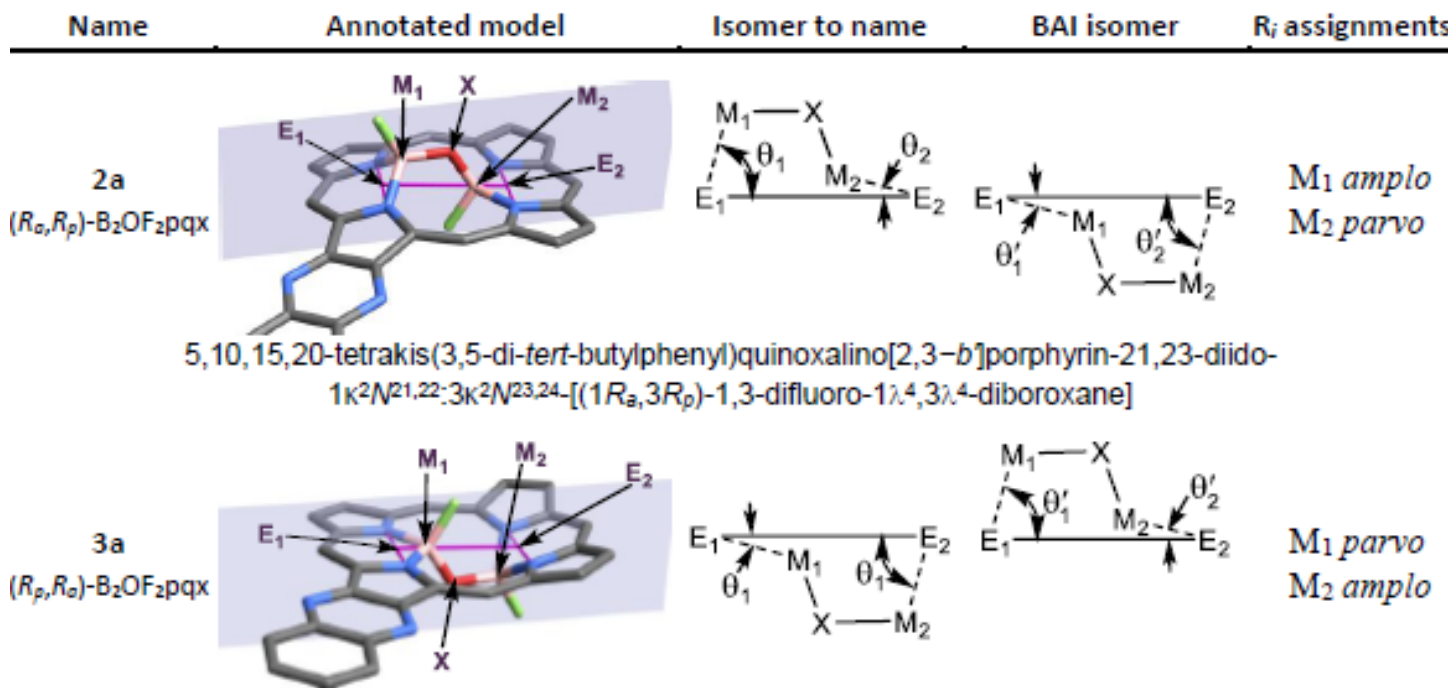

5,10,15,20-tetrakis(3,5-di-tert-butylphenyl)quinoxalino[2,3- $\left.b^{7}\right]$ porphyrin-21,23-diido-

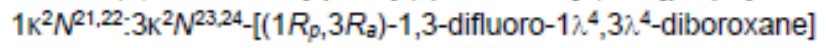

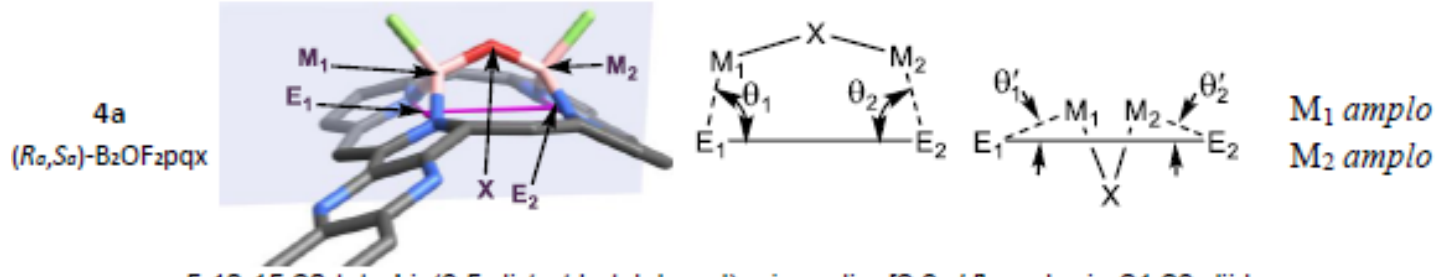

5,10,15,20-tetrakis (3,5-di-tert-butylphenyl)quinoxalino[2,3-b']porphyrin-21,23-diido$1 \mathrm{~K}^{2} N^{21,22-3 K^{2}} N^{23,24-[}\left[\left(1 R_{a}, 3 S_{a}\right)-1,3\right.$-difluoro-1 $14^{4}, 3 \lambda^{4}$-diboroxane]

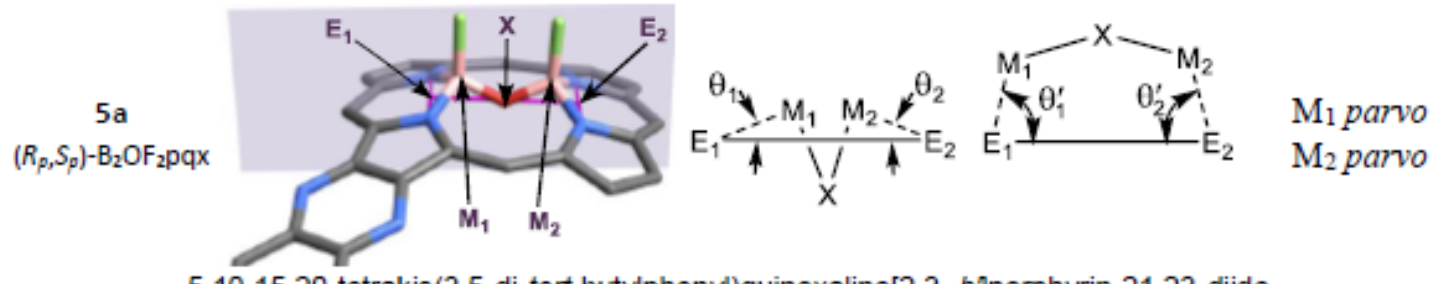

5,10,15,20-tetrakis (3,5-di-tert-butylphenyl)quinoxalino[2,3-b]porphyrin-21,23-diido$1 \mathrm{~K}^{2} N^{21,22-3 K^{2}} N^{23,24}-\left[\left(1 R_{p}, 3 S_{p}\right)-1,3\right.$-difluoro-1 $1{ }^{4}, 3 \lambda^{4}$-diboroxane $]$

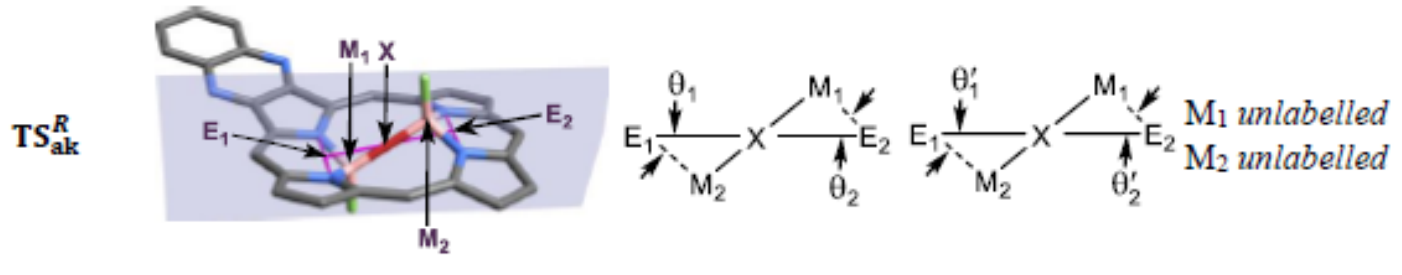

5,10,15,20-tetrakis(3,5-di-tert-butylphenyl)quinoxalino[2,3-b]porphyrin-21,23-

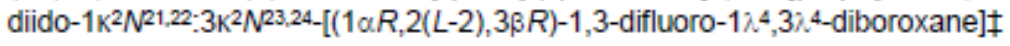

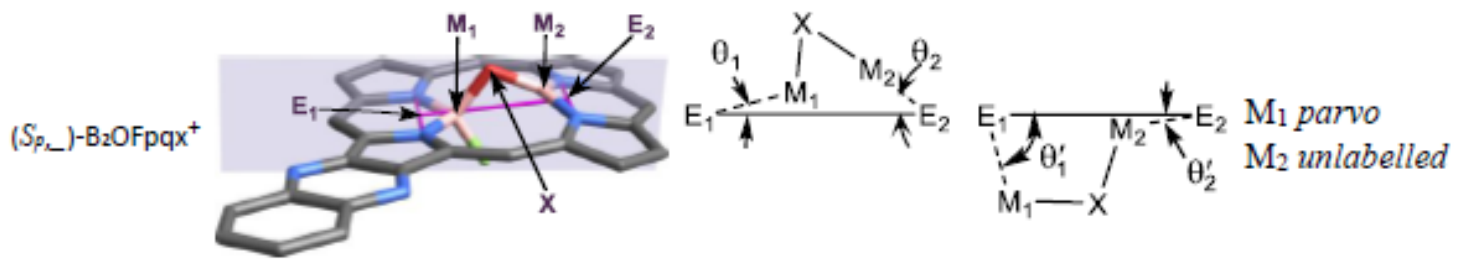

5,10,15,20-tetrakis (3,5-di-tert-butylphenyl)quinoxalino[2,3- $b$ ]porphyrin-21,23-diido-

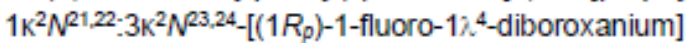

